\title{
GAD autoantibody affinity in schoolchildren from the general population
}

\author{
Christine Bender • Michael Schlosser • Urs Christen • \\ Anette G. Ziegler • Peter Achenbach
}

Received: 10 January 2014 / Accepted: 16 May 2014 / Published online: 18 June 2014

(C) Springer-Verlag Berlin Heidelberg 2014

\begin{abstract}
Aims/hypothesis Subtyping GAD autoantibody (GADA) responses using affinity measurement allows the identification of GADA-positive children with a family history of type 1 diabetes who are at risk of developing diabetes. Here, we asked whether GADA affinity is a useful marker to stratify the risk of type 1 diabetes in GADA-positive schoolchildren from the general population.

Methods GADA affinity was measured by competitive binding experiments with $\left.{ }^{125} \mathrm{I}\right]$-labelled and unlabelled human $65 \mathrm{kDa}$ isoform of GAD (GAD65) in sera from 97 GADApositive children identified in the Karlsburg Type 1 Diabetes Risk Study of a general schoolchild population in northeastern Germany. GADA epitope specificity was determined using radiobinding assays with $\left[{ }^{35} \mathrm{~S}\right]$-labelled GAD65/67 kDa isoform of GAD (GAD67) chimeric proteins.

Results GADA affinity was high, $\geq 10^{10} 1 / \mathrm{mol}$, in 33 of 35 multiple islet autoantibody-positive children. In contrast, the affinity ranged widely among 62 single GADA-positive
\end{abstract}

C. Bender $(\bowtie) \cdot U$. Christen

Pharmazentrum Frankfurt/ZAFES, Goethe University Hospital

Frankfurt, Theodor-Stern Kai 7, 60590 Frankfurt am Main, Germany

e-mail: bender@med.uni-frankfurt.de

M. Schlosser

Department of Medical Biochemistry and Molecular Biology,

Research Group of Predictive Diagnostics, and Institute of

Pathophysiology, Research Group of Predictive Diagnostics,

University Medical Center Greifswald, Karlsburg, Germany

A. G. Ziegler • P. Achenbach $(\bowtie)$

Institute of Diabetes Research, Helmholtz Zentrum München, Ingolstaedter Landstrasse 1, 85764 Neuherberg, Germany

e-mail: peter.achenbach@helmholtz-muenchen.de

A. G. Ziegler • P. Achenbach

Forschergruppe Diabetes, Klinikum rechts der Isar, Technische

Universität München, Munich, Germany children (median $3.1 \times 10^{9} \mathrm{l} / \mathrm{mol}$; range $5.6 \times 10^{6}$ to $>4.0 \times$ $\left.10^{11} \mathrm{l} / \mathrm{mol} ; p<0.0001\right)$. High-affinity GADA were associated with HLA-DRB ${ }^{*} 03(p=0.02)$ and predominantly directed against the C-terminal and/or middle part of the GAD65 protein. At follow-up, the affinity remained relatively constant. Five of the single GADA-positive children developed additional islet autoantibodies and had high-affinity GADA. Twenty-six children progressed to type 1 diabetes; among them, 23 had GADA affinities of $\geq 10^{10} 1 / \mathrm{mol}$ before disease onset.

Conclusions/interpretation Schoolchildren from the general population may develop heterogeneous GADA responses, and a high affinity can identify those GADA-positive children who are more likely to progress to type 1 diabetes.

Keywords Affinity · GAD autoantibodies · General population $\cdot$ Schoolchildren $\cdot$ Type 1 diabetes risk

$\begin{array}{ll}\text { Abbreviations } \\ \text { GAD65 } & \text { 65 kDa isoform of GAD } \\ \text { GAD67 } & \text { 67 kDa isoform of GAD } \\ \text { GADA } & \text { Autoantibodies to GAD } \\ \text { GAD-MID } & \text { Middle section of GAD } \\ \text { IA-2A } & \begin{array}{l}\text { Autoantibodies to insulinoma-associated } \\ \text { antigen-2 }\end{array} \\ \text { IAA } & \text { Autoantibodies to insulin } \\ \text { IQR } & \text { Interquartile range } \\ \text { ZnT8A } & \text { Autoantibodies to zinc transporter-8 }\end{array}$

\section{Introduction}

Type 1 diabetes is one of the most common autoimmune diseases in children and adolescents and is caused by a selective destruction of the insulin-producing beta cells of the 
pancreatic islets of Langerhans [1,2]. A characteristic feature of this disease is the occurrence of autoantibodies against beta cell antigens such as autoantibodies to GAD (GADA), insulin (IAA), insulinoma-associated antigen-2 (IA-2A) and zinc transporter-8 (ZnT8A) [3, 4]. The presence of such autoantibodies in the peripheral blood is the first detectable marker of ongoing beta cell destruction. They may appear early in life and predict the development of type 1 diabetes [5-8]. The risk of diabetes increases with the number of autoantibody markers $[9,10]$. In contrast, children who do not develop autoantibodies against multiple beta cell antigens rarely progress to clinical type 1 diabetes during childhood. GADA often appear in older age groups and are preferentially associated with HLA-DRB $1 * 03$ haplotypes [11]. The GADA level alone does not predict progression to diabetes, and controversial reports exist with respect to the relevance of GADA epitope reactivity to diabetes progression [12-17].

We previously demonstrated that children with a familial predisposition who developed type 1 diabetes had highaffinity GADA before the onset of their disease, whereas those with low-affinity GADA showed no progression of islet autoimmunity [14]. Nevertheless, approximately $90 \%$ of children diagnosed with type 1 diabetes have no first-degree relatives with the disease [18-20]. The intent of the present study was therefore to extend our previous findings in relatives to schoolchildren from the general population. In particular, we asked whether a relationship exists between GADA affinity and other characteristics of GADA such as level and epitope specificity, as well as HLA genotype and the status of autoantibodies to other beta cell antigens.

\section{Methods}

Study cohort and samples Samples were obtained from the Karlsburg Type 1 Diabetes Risk Study, in which a total of 11,840 children of a general school population in the northeastern part of Germany were screened for GADA, IAA, IA2A and islet cell antibodies [21]. Autoantibody-positive children were invited for re-examination to confirm the autoantibody measurements and for HLA genotyping [22]. The study was authorised by the state government of MecklenburgVorpommern (No. VII 233 A-3201-01) and approved by the ethics committee of the University of Greifswald. All participants gave written informed consent to participate in the study. Investigations were carried out in accordance with the principles of the Declaration of Helsinki, as revised in 2008.

For the present study, the sample volume of 97 GADApositive schoolchildren at re-examination was large enough to perform measurements of GADA affinity and epitopes. This sample was defined as the initial sample in our study. The children had a median age of 11.2 years (interquartile range [IQR] 9.6-13.5 years), 44 were male, and none had a first- degree relative with type 1 diabetes. In addition, a follow-up sample was available for analysis from 95 children (median time since the initial sample 4.9 years, IQR 3.3-6.6 years), and a second follow-up sample from 13 children (median time since the first follow-up sample 3.7 years, IQR 2.1-4.4 years). The children were contacted on follow-up to obtain information on diabetes status (median time from the initial sample to last contact 8.6 years, IQR $5.2-10.9$ years).

Islet autoantibody measurements Levels of GADA, IA-2A and IAA were measured by radiobinding assays using $\left[{ }^{125} \mathrm{I}\right]-$ labelled recombinant human $65 \mathrm{kDa}$ isoform of $\mathrm{GAD}$ (GAD65), IA-2 and insulin as previously described [20, 21]. A cut-off level at or above the 98th percentile was used for all autoantibodies [21]. In the 4th Diabetes Antibody Standardization Program 2005 workshop, the GADA and IA-2A assays achieved a diagnostic sensitivity and specificity of $82 \%$ and $96 \%$ (GADA) and $66 \%$ and $100 \%$ (IA-2A), respectively [23], and the IAA assay achieved $58 \%$ sensitivity and $97 \%$ specificity [24].

GADA epitope measurements GADA epitope specificity was determined using radiobinding assays on $\left[{ }^{35} \mathrm{~S}\right]$-methioninelabelled GAD65/67 kDa isoform of GAD (GAD67) chimeric proteins as previously described [17]. Thresholds for positivity were defined as the upper limit in the sera of 50 control participants. The chimeric proteins used were GAD65 ${ }_{1-95} /$ GAD67 101-593 (GAD N-terminal; GAD65 amino acid 1-95), GAD67 $_{1-243} /$ GAD65 $_{235-444} /$ GAD67 $_{453-593}$ (GAD-MID, the middle section of GAD; GAD65 amino acid 235-444) and

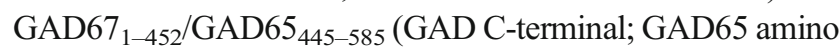
acid 445-585). GADA were classified as A (reactive to middle and/or C-terminal GAD65 epitopes), B (reactive to N-terminal GAD65 epitopes only) or C (reactive to none of the GAD65 epitopes represented in the chimeric proteins).

GADA affinity measurements GADA affinity was measured by competitive binding experiments as previously described [14]. Briefly, serum $(2 \mu \mathrm{l})$ was incubated in duplicate for $48 \mathrm{~h}$ at $4^{\circ} \mathrm{C}$ in TBST buffer $(50 \mathrm{~mol} / \mathrm{l}$ Tris, $150 \mathrm{mmol} / \mathrm{l} \mathrm{NaCl}, 1 \%$ Tween 20, pH 7.4) in the presence of $\left[{ }^{125} \mathrm{I}\right]$-recombinant human GAD65 $(0.05 \mathrm{nmol} / \mathrm{l})$ and five increasing quantities of unlabelled human GAD65 $\left(3.3 \times 10^{-11}, 3.3 \times 10^{-10}, 3.3 \times\right.$ $10^{-9}, 3.3 \times 10^{-8}$ and $3.3 \times 10^{-7} \mathrm{~mol} / \mathrm{l}$ ) (RSR Ltd., Cardiff, UK) or TBST buffer only in a final volume of $47 \mu$ l. Immune complexes were precipitated for $60 \mathrm{~min}$ with $1.5 \mathrm{mg}$ Protein A Sepharose (GE Healthcare, Chalfont St Giles, UK) preswelled in $50 \mu \mathrm{TBST}$, subsequently washed five times with $1.8 \mathrm{ml}$ of ice-cold TBST buffer and measured using a gamma counter (Packard Instruments Co., Meriden, CT, USA). The results were expressed as cpm.

$\mathrm{IC}_{50}$ and $K_{\mathrm{d}}$ values were calculated by non-linear regression analysis using GraphPad Prism3 (GraphPad Software, 
San Diego, CA, USA), and the mean GADA affinity of each sample was expressed as the reciprocal $K_{\mathrm{d}}$ value $(1 / \mathrm{mol})$ (Fig. 1). Samples with very high GADA levels that showed binding to $\left[{ }^{125} \mathrm{I}\right]$-GAD 65 of $>50 \%$ of the total $\left[{ }^{125} \mathrm{I}\right]$-GAD 65 activity added to the test were diluted. For such samples, GADA affinity was calculated from the competitive binding curve obtained with the diluted sample. The reproducibility of GADA affinity measurements was determined from replicates of a GADA-positive serum that was included in each experiment. The CV of GADA affinity measurements was $3 \%$ [14]. The calculation of $K_{\mathrm{d}}$ values was limited to samples with $\mathrm{IC}_{50}$ values greater than the concentration of labelled GAD65 $(0.05 \mathrm{nmol} / \mathrm{l})$. For samples with an $\mathrm{IC}_{50}<0.05 \mathrm{nmol} / \mathrm{l}$, the mean GADA affinity of the sample was set at $>4.0 \times 10^{11} \mathrm{l} / \mathrm{mol}$.

HLA typing HLA-DRB1 genotyping was performed using PCR-amplified genomic DNA and nonradioactive oligonucleotide probes as described elsewhere [22].

Statistical analysis The Mann-Whitney $U$ test was used to compare GADA affinities between groups (epitope specificity, autoantibody status and HLA genotype). Spearman's correlation was used to determine the correlation between variables. The Wilcoxon logrank test was used to compare GADA affinity over time. For all analysis, a two-tailed $p$ value of 0.05 was considered significant. All statistical analyses were performed using GraphPad Prism software (Version 3.01; GraphPad Software).

\section{Results}

Schoolchildren from the general population exhibit large variations in GADA affinity The affinity of GADA varied between the 97 GADA-positive schoolchildren and ranged from $5.6 \times 10^{6}$ to $>4.0 \times 10^{11} \mathrm{l} / \mathrm{mol}$. Competitive GADA binding curves to $\left[{ }^{125} \mathrm{I}\right]$-GAD65 were consistent with a one-site binding model in the samples from all the children, suggesting that GADA were of relatively homogeneous affinities within each sample (Fig. 1). The affinity of GADA correlated with the GADA level $(r=0.57 ; p<0.0001)$ but was not associated with age $(p=0.2)$. Children carrying the HLA-DRB $1 * 03$ allele showed higher GADA affinities $\left(n=28\right.$; median $>4.0 \times 10^{11}$ $1 / \mathrm{mol}$, IQR $1.3 \times 10^{10}$ to $>4.0 \times 10^{11} \mathrm{l} / \mathrm{mol}$ ) compared with those with other HLA alleles $\left(n=69\right.$; median $1.4 \times 10^{10}$ $\mathrm{l} / \mathrm{mol}$, IQR $8.2 \times 10^{8}$ to $>4.0 \times 10^{11} \mathrm{l} / \mathrm{mol} ; p=0.02$ ) (Fig. $2 \mathrm{a}$ ). GADA affinity was particularly higher in children with coexisting IAA and/or IA-2A $\left(n=35\right.$; median $>4.0 \times 10^{11}$ $\mathrm{l} / \mathrm{mol}$, IQR $3.9 \times 10^{11}$ to $>4.0 \times 10^{11} \mathrm{l} / \mathrm{mol}$ ) compared with those with GADA alone $\left(n=62\right.$; median $3.1 \times 10^{9} 1 / \mathrm{mol}$, IQR $6.2 \times 10^{8}$ to $1.8 \times 10^{11} 1 / \mathrm{mol} ; p<0.0001$ ) (Fig. $2 \mathrm{~b}$ ).

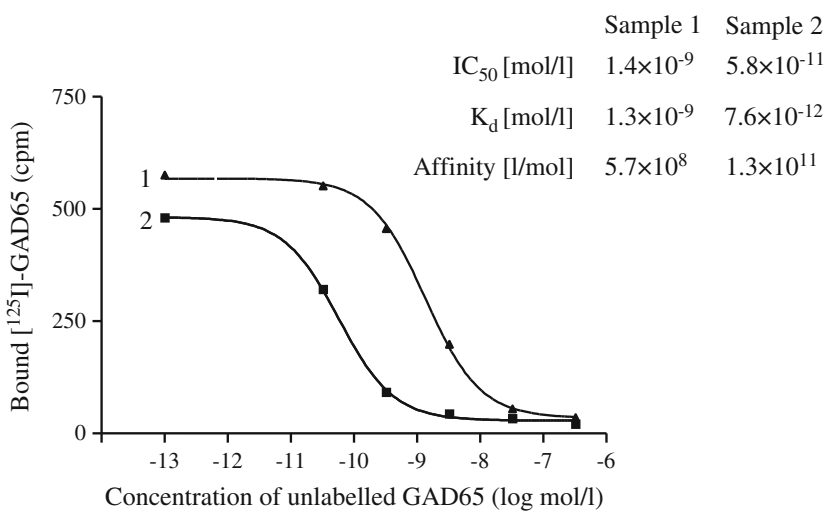

Fig. 1 Sample competitive binding curves. GADA binding to human $\left[{ }^{125} \mathrm{I}\right]$-GAD65 ( $y$-axis) at increasing concentrations of competitive unlabelled human GAD65 ( $x$-axis; $\log _{10}$ transformed values) is shown for a serum with low-affinity GADA of $5.7 \times 10^{8} 1 / \mathrm{mol}$ (sample 1 ) and a serum with high-affinity GADA of $1.3 \times 10^{11} \mathrm{l} / \mathrm{mol}$ (sample 2 ). Both curves are consistent with a one-site binding model

High-affinity GADA display a characteristic epitope specificity The GADA response was predominantly directed against the C-terminal (residues 445-585) and/or middle part (residues 235-444) of the GAD65 protein. This epitope reactivity was associated with high GADA affinities $(n=70$; median $>4.0 \times 10^{11} \mathrm{l} / \mathrm{mol}$, IQR $4.4 \times 10^{9}$ to $>4.0 \times 10^{11} \mathrm{l} / \mathrm{mol}$ ) compared with GADA responses restricted to the $\mathrm{N}$-terminal part (residues 1-95) of the protein, which were associated with lower affinities $\left(n=12\right.$; median $9.0 \times 10^{8} \mathrm{l} / \mathrm{mol}$, IQR $2.1 \times 10^{8}$ to $2.9 \times 10^{9} \mathrm{l} / \mathrm{mol} ; p<0.0001$ ) (Fig. $2 \mathrm{c}$ ). Furthermore, GADA levels correlated well with GADA reactivity to the $\mathrm{C}$-terminal $(r=0.73 ; p<0.0001)$ and middle $(r=0.77 ; p<0.0001)$ part of GAD65 (Fig. 3a, b), but not with GADA reactivity to the N-terminal region $(r=0.07 ; p=0.5)$ (Fig. $3 \mathrm{c}$ ). GADA of unknown epitope specificity (i.e. GADA that did not bind to the GAD-MID, C-terminal, or N-terminal constructs) showed variable affinities (median $1.5 \times 10^{10} \mathrm{l} / \mathrm{mol}$, IQR $7.7 \times 10^{8}$ to $>4.0 \times 10^{11} 1 / \mathrm{mol}$ ) (Fig. 2c).

GADA affinity on follow-up and progression to type 1 diabetes We obtained a first follow-up serum sample from 95 of the 97 GADA-positive schoolchildren, and a second follow-up sample from 13 children. No follow-up sample was available from two multiple islet autoantibody-positive children (GADA affinity $3.1 \times 10^{10}$ and $>4.0 \times 10^{11} \mathrm{l} / \mathrm{mol}$, respectively). A total of 26 children developed type 1 diabetes (median time from initial sample to diagnosis 6.5 years, IQR $2.8-10.6$ years).

GADA affinity remained relatively constant on follow-up in the majority of children (Fig. 4). Children with multiple islet autoantibodies $(n=33)$ predominantly had GADA affinities of $\geq 10^{10} \mathrm{l} / \mathrm{mol}$. Of these, 29 children remained multiple autoantibody-positive on follow-up, and 21 of them developed diabetes. Four children reverted to single GADA, but two of them nevertheless developed diabetes (Fig. 4c, d). In 
a

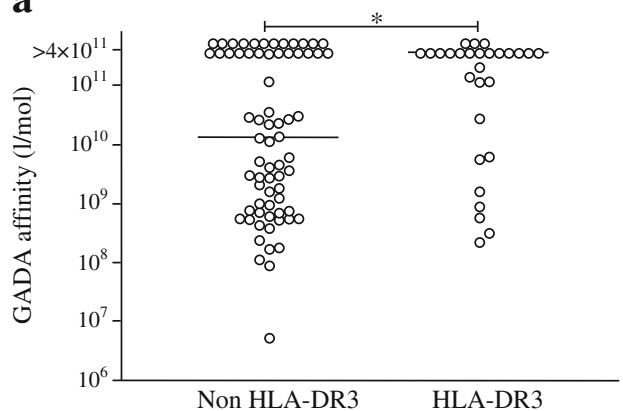

b
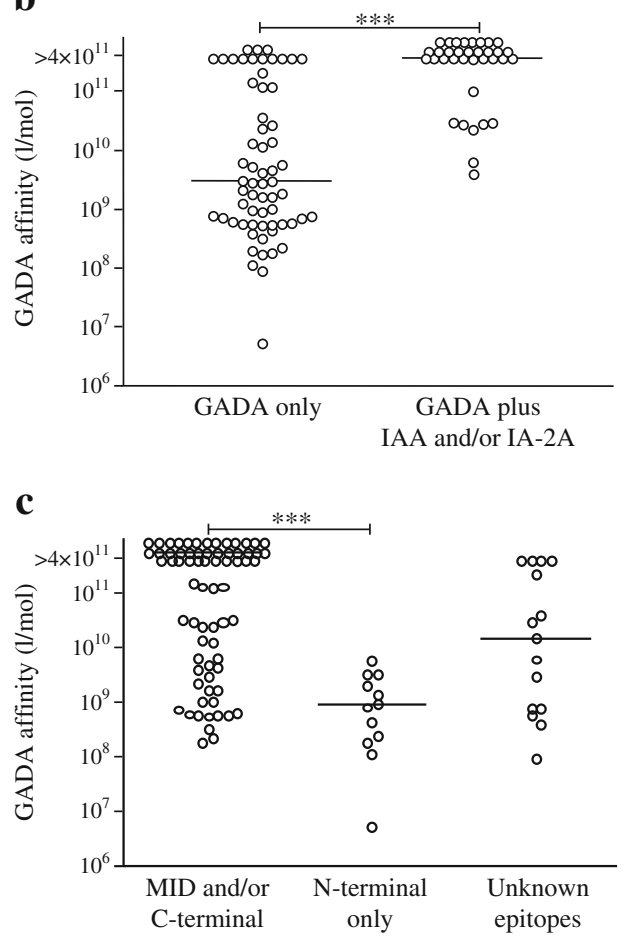

Fig. 2 Relationship between GADA affinity and HLA genotype, islet autoantibody status or GADA epitope binding. The GADA affinities of 97 schoolchildren are plotted and compared between (a) children with the HLA DRB $1 * 03$ allele (HLA-DR3; $n=28$ ) vs those without this allele (non-HLA-DR3; $n=69$ ); (b) children with GADA only $(n=62)$ vs those with GADA plus IAA and/or IA-2A $(n=35)$; and (c) children with GADA binding to epitopes within GAD65 residues 235-585 (MID and/or C-terminal; $n=70)$, restricted binding to epitopes within GAD65 residues $1-95$ (N-terminal only; $n=12$ ) or binding to unknown epitopes (MID-, C-, N-terminal negative; $n=15$ ). The median GADA affinity is indicated for each group. ${ }^{*} p<0.05,{ }^{* * *} p<0.001$

contrast, children with single GADA $(n=62)$ showed a broad spectrum of GADA affinities ranging from $10^{6}$ to $10^{11} \mathrm{l} / \mathrm{mol}$. Among these, 57 children remained single GADA-positive on follow-up, and two developed diabetes. Five children became multiple islet autoantibody-positive on follow-up (GADA affinity, range $1.2 \times 10^{10}$ to $>4.0 \times 10^{11} \mathrm{l} / \mathrm{mol}$ ), and one developed diabetes (Fig. 4a, b).

GADA affinity, level and epitope specificity define distinct GADA profiles On considering the affinity, level and epitope reactivity of GADA, as well as islet autoantibody status and HLA genotype, several distinct GADA profiles could be identified to stratify the risk of type 1 diabetes in schoolchildren (Fig. 5).

The first, most frequent GADA profile (profile P1; Fig. $5 \mathrm{a}, \mathrm{d})$ was found in $50(51.5 \%)$ of the 97 GADApositive children. This profile displayed high-affinity GADA with median to high antibody levels and reactivity against the middle and/or C-terminal epitopes of the GAD65 protein. Of the children with this profile, $20(40 \%)$ carried the HLADRB1*03 allele, $31(62 \%)$ were multiple islet autoantibodypositive, and 23 (46\%) developed type 1 diabetes.

A second GADA profile (profile P2; Fig. 5a) was also associated with reactivity against the middle and/or C-terminal regions of GAD65, but showed low GADA affinity and low antibody levels. This profile was found in 20 (20.6\%) of the 97 children who were all single GADA-positive. Only four of them $(20 \%)$ carried the HLA-DRB $1 * 03$ allele, and one (5\%) progressed to type 1 diabetes.

The third GADA profile (profile P3; Fig. 5b, e) was characterised by restricted GADA binding against the N-terminal epitopes of GAD65, low GADA affinity, low antibody levels and low frequency of the HLA-DRB $1 * 03$ allele. Twelve (12.4\%) children displayed this profile, of whom 11 were single GADA-positive and none progressed to type 1 diabetes.

Finally, 15 (15.5\%) children had GADA that did not bind to any of the epitope constructs (profile P4; Fig. 5c, f). These GADA were of either low or high affinity, had low antibody levels and were found in children with single GADA or multiple islet autoantibodies. Only two children with this fourth GADA profile progressed to type 1 diabetes (both of whom were multiple autoantibody-positive).

A summary of the main characteristics of all the GADA profiles is shown in Fig. $5 \mathrm{~g}$. Among multiple islet autoantibody-positive children, profile P1 was the predominant GADA profile ( $88.6 \%$; Fig. 5j), whereas single GADApositive children showed a rather variable distribution into the four profiles (Fig. 5h).

\section{Discussion}

Screening for autoantibodies to beta cell antigens is a powerful method to assess the risk of type 1 diabetes [10]. It has recently become evident that besides the autoantibody level, both affinity and epitope specificity may be used to further stratify risk $[12,14,25,26]$. We were previously able to demonstrate in children who had at least one parent affected by type 1 diabetes that an assessment of GADA affinity and epitope specificity allowed us to define discrete GADA profiles associated with risk of type 1 diabetes [14]. The present study is 


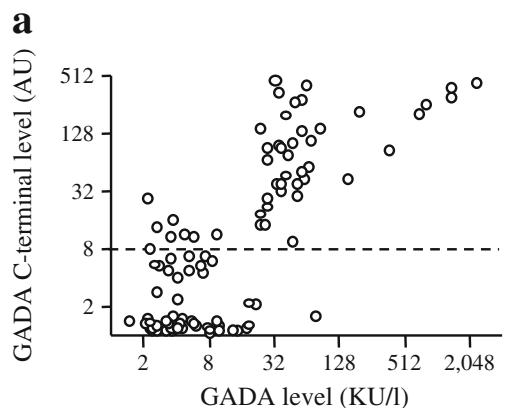

Fig. 3 Relationship between GADA levels and epitope binding. The GADA levels of 97 schoolchildren are correlated with the levels of GADA binding to epitopes within (a) GAD65 residues 445-585 (GADA C-terminal) $(r=0.73, p<0.0001)$, (b) GAD65 residues 235-444 (GADA-

the first to expand this observation to the general population and to analyse GADA profiles in schoolchildren without a first-degree family history of type 1 diabetes. As in the relatives [14], we found GADA profiles that were characterised by their GADA affinity and epitope specificity. In particular, children with high-affinity GADA that recognised the middle and/or C-terminal part of GAD65 (profile P1) were at risk of progression to clinical diabetes. IAA and/or IA-2A as well as the HLA-DRB $1 * 03$ allele were frequently present in GADApositive children with this profile.

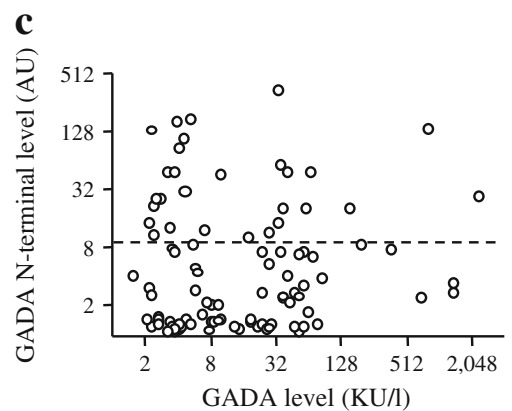

MID) $(r=0.77, p<0.0001)$, or (c) GAD65 residues 1-95 (GADA N-terminal) $(r=0.07, p=0.5)$. Thresholds for antibody positivity are indicated by broken lines for each epitope specificity. AU, arbitrary units; KU, Karlsburg units

The affinity of GADA varied among the schoolchildren over almost five orders of magnitude and was correlated with GADA levels. Interestingly, GADA binding to the middle and/or C-terminal part of GAD65 could be of high or low affinity, and this was strongly related to the islet autoantibody status. The occurrence of GADA plus IAA and/or IA-2A was predominantly seen in children with high-affinity autoantibodies, confirming previous reports from us and others [25, $27,28]$. In contrast, GADA binding only to the N-terminal part of GAD65 involved predominantly single low-affinity
Fig. 4 GADA affinity over time in 203 follow-up samples from 95 schoolchildren. GADA affinity on follow-up is shown for 62 children with GADA only $(\mathbf{a}, \mathbf{b})$ and 33 children with GADA plus IAA and/or IA-2A (c, d) in their first serum samples. Follow-up samples from individual children are connected by lines. Multiple islet autoantibody-positive sera are indicated by filled circles. Children who progressed to type 1 diabetes (T1D) on follow-up $(n=26)$ are presented separately (b, d) a
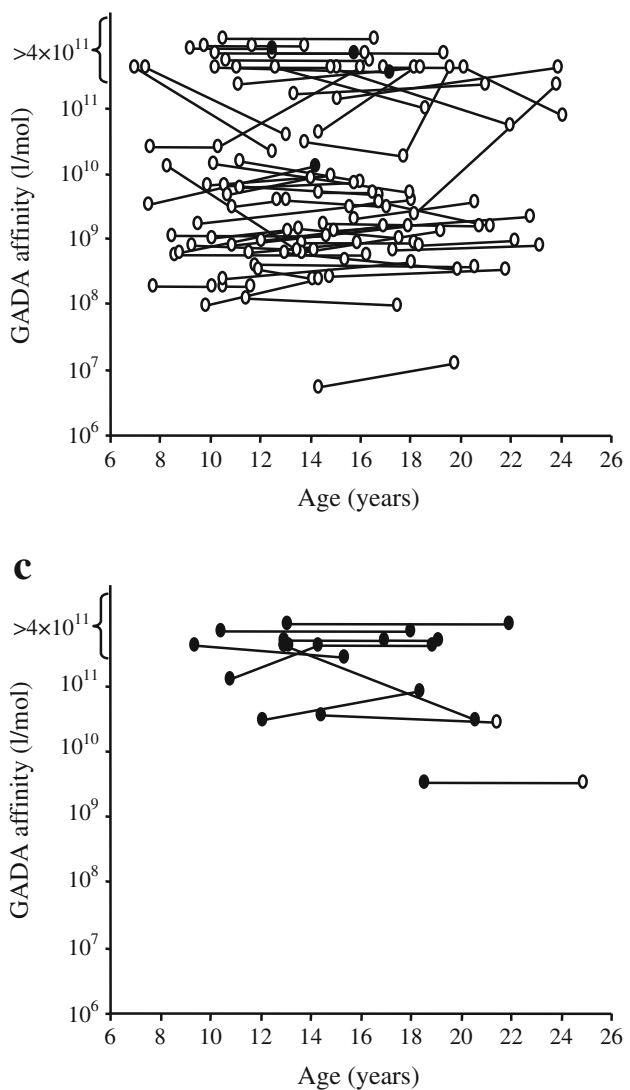

b

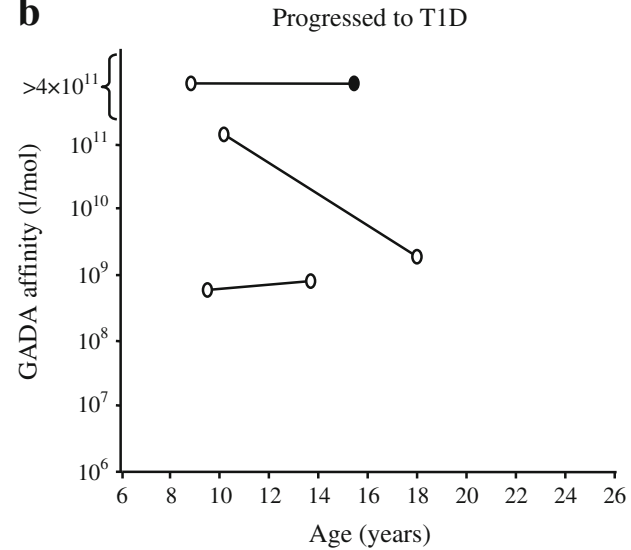

d

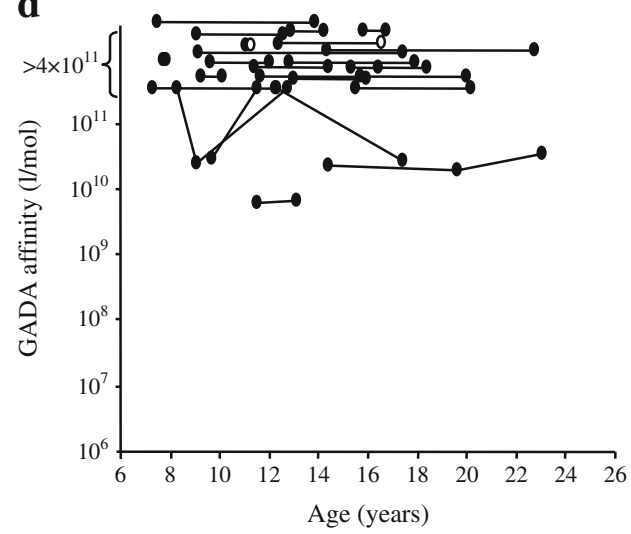


Fig. 5 GADA profiles. Sera from 97 schoolchildren are grouped according to islet autoantibody status: GADA only $(\mathbf{a}, \mathbf{b}, \mathbf{c})$, or GADA plus IAA and/or IA-2A (d, e, f). GADA epitope reactivity: GAD65 middle and/or C-terminal epitopes (MID and/or C-terminal) (a, d); GAD65 $\mathrm{N}$-terminal epitopes only (N-terminal only) (b, e); or unknown GAD65 epitope specificity (MID-, C-, N-terminal negative) (c, f). Sera with similar GADA profiles are circled (profiles P1-P4). Children with allele HLA-DRB $1 * 03$ are indicated by filled triangles $(\mathbf{a}-\mathbf{f})$. The table summarises the characteristics of the GADA profiles (g). The frequency of profiles is shown for children with GADA only (h) or GADA plus IA-2A and/or IAA (j). KU, Karlsburg units
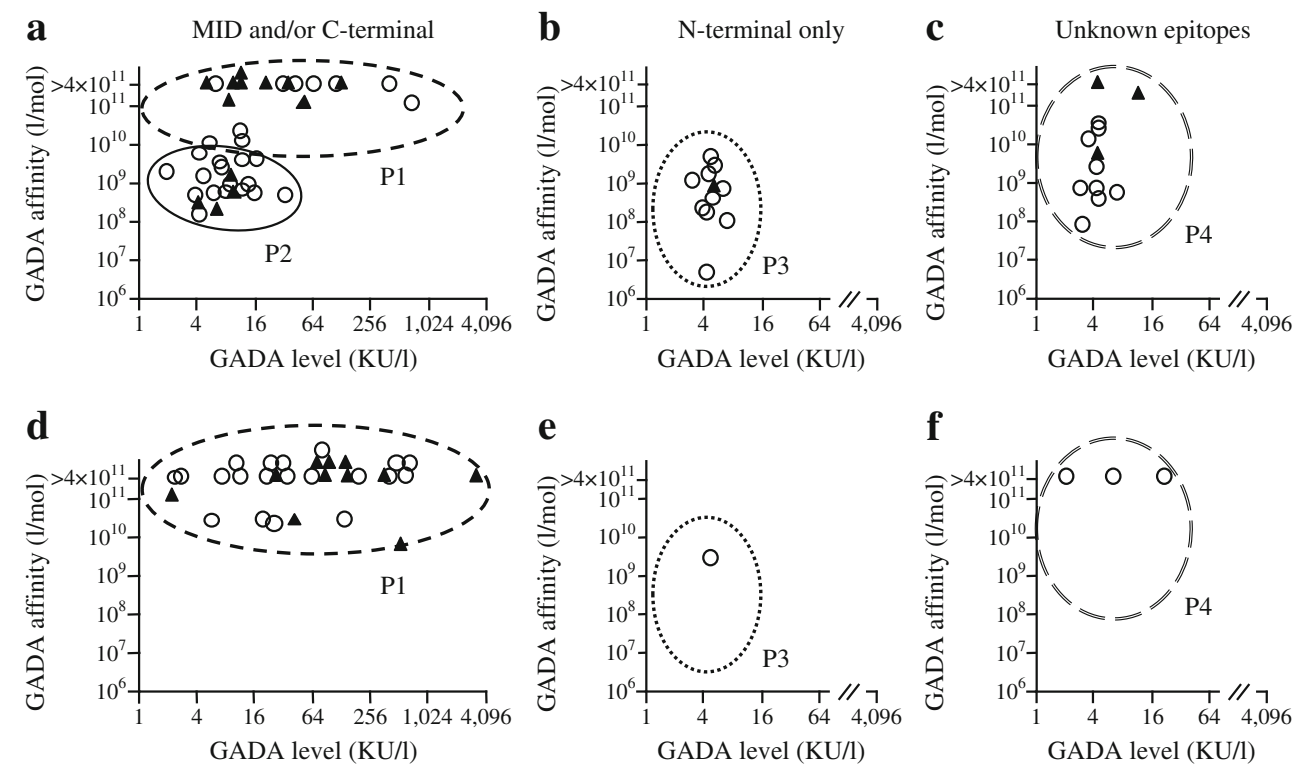

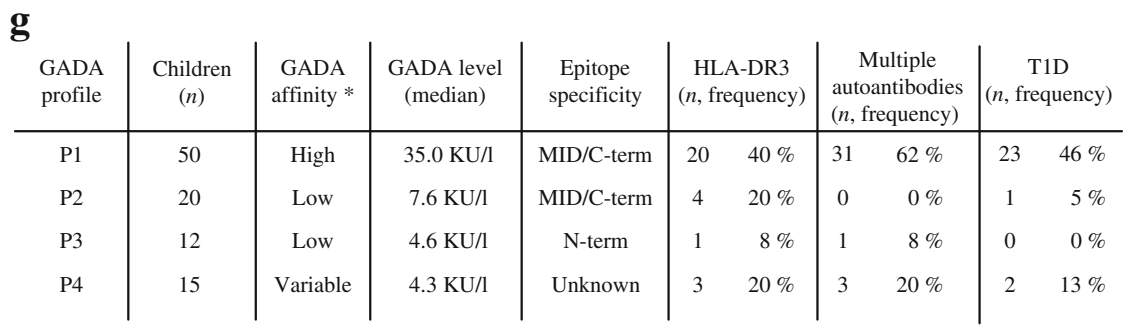

GADA affinity: high $>10^{10} \mathrm{l} / \mathrm{mol}$; low $<10^{10} \mathrm{l} / \mathrm{mol}$
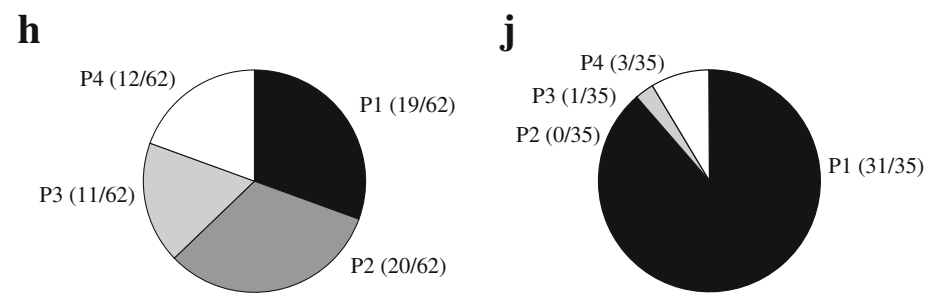

antibodies and was not related to the development of diabetes. Overall, low-affinity GADA were rarely found in multiple autoantibody-positive children.

GADA profiles were relatively homogenous in schoolchildren with multiple islet autoantibodies and those who developed diabetes, but were highly variable in children who had only GADA. Moreover, children with single lowaffinity GADA could be classified into two profiles according to their binding to epitopes within the middle/C-terminal region of GAD65 (profile P2) or within the $\mathrm{N}$-terminal region only (profile P3). Besides the absence of reactivity to multiple islet autoantigens, both profiles were further characterised by relatively low GADA levels and a low frequency of the HLA$\mathrm{DRB} 1 * 03$ allele. Only one child displaying profile $\mathrm{P} 2$, and no child with profile $\mathrm{P} 3$, developed type 1 diabetes during the observation time. These findings in the GADA-positive children from the general population are consistent with what we and others have previously observed for GADA, IAA or IA$2 \mathrm{~A}$ in first-degree relatives and in IAA-positive children without a familial predisposition [14, 25, 26, 28, 29].

A limitation of our study is that, in contrast to the BABYDIAB study [8], only sera from schoolchildren with established GADA responses have been included, and the age at autoantibody seroconversion in these children was not known. This may explain the low number of changes from single GADA to multiple autoantibody-positive status. Only a few single GADA-positive children developed IAA and/or IA-2A on follow-up. Another limitation of our study is that the schoolchildren were not tested for the presence of ZnT8A. Therefore, we cannot exclude the possibility that some of the schoolchildren who were classified as single GADA-positive may have had ZnT8A and would shift to the multiple islet autoantibody-positive group. ZnT8A have been associated with rapid disease progression in the relatives of patients with 
type 1 diabetes [30-32], which we did not observe for schoolchildren with GADA only in the present study. Yet the selected cohort of schoolchildren in our study allowed for an analysis of the development of GADA affinity and epitope specificity over time. Since the children are still being followed prospectively into adulthood, we cannot identify the timeframe within which children with a specific GADA profile will progress to clinical diabetes. Nevertheless, our findings confirm immune profiles found in the relatives of type 1 diabetes patients [14]. Therefore, the data support the hypothesis that findings related to the immune pathogenesis of type 1 diabetes identified in people with a familial predisposition can be transferred to the general population. Recent advances in the reliability and specificity of islet autoantibody measurements, such as electrochemiluminescence-based antibody assays [33], may further improve screening in the general population.

In summary, we conclude that an assessment of GADA affinity and epitope specificity in addition to the GADA level, islet autoantibody status and HLA genotype allows for the determination of several distinct GADA profiles and a more accurate stratification of the risk of type 1 diabetes in the general population. Furthermore, GADA affinity could be a useful diagnostic marker for the recruitment of people from the general population to studies on the prevention of type 1 diabetes.

Acknowledgements The authors thank B. Rees-Smith (RSR, Cardiff, UK) for providing recombinant human GAD65, R. Wassmuth (Department of Quality Management, University Medical Center, University of Duesseldorf, Germany) for HLA genotyping and S. Krause (Forschergruppe Diabetes, Klinikum rechts der Isar, Technische Universität München, Germany) for expert technical assistance.

Funding This study was supported in part by grants from the European Union (EP7-HEALTH-2007, DIAPREPP N202013) and from the German Federal Ministry of Education and Research to the Competence Network for Diabetes mellitus (FKZ 01GI0805) and to the German Center for Diabetes Research (DZD e.V.). PA received support from the JDRF (11-2005-1117).

The Karlsburg Type 1 Diabetes Risk Study was supported by the German Federal Ministry of Research and Technology (BMFT 07NBL02/D4), the government of Mecklenburg-Vorpommern (EMAU16/1995), the Else-Kröner-Fresenius-Stiftung, the Deutsche Diabetes-Stiftung, the Community Medicine Project of the Ernst Moritz Arndt University Greifswald, and the BRAHMS Diagnostica GmbH, Berlin. Additional support was obtained from the BMFT-funded Center for Interdisciplinary Clinical Research at the Friedrich Alexander University Erlangen-Nuernberg (01KS9601), the Deutsche Forschungsgemeinschaft (SFB263), and the Association for Support of Diabetes Research, Karlsburg, Greifswald e.V.

Duality of interest The authors declare that there is no duality of interest associated with this manuscript.

Contribution statement $\mathrm{CB}$ performed all the experiments, researched data and wrote, reviewed and edited the manuscript. MS and UC contributed to the writing of the manuscript. PA designed and supervised the study, researched data and reviewed and edited the manuscript. All authors (CB, MS, UC, AGZ and PA) contributed to the interpretation of data, critically revised the manuscript for important intellectual content, and approved the final version of the manuscript. PA takes responsibility for the integrity of the work as a whole.

\section{References}

1. Bluestone JA, Herold K, Eisenbarth G (2010) Genetics, pathogenesis and clinical interventions in type 1 diabetes. Nature 464:1293-1300

2. Castano L, Eisenbarth GS (1990) Type-I diabetes: a chronic autoimmune disease of human, mouse, and rat. Annu Rev Immunol 8:647-679

3. Stadinski B, Kappler J, Eisenbarth GS (2010) Molecular targeting of islet autoantigens. Immunity 32:446-456

4. Wenzlau JM, Juhl K, Yu L et al (2007) The cation efflux transporter $\mathrm{ZnT} 8$ (Slc30A8) is a major autoantigen in human type 1 diabetes. Proc Natl Acad Sci U S A 104:17040-17045

5. Atkinson MA, Eisenbarth GS (2001) Type 1 diabetes: new perspectives on disease pathogenesis and treatment. Lancet 358:221-229

6. Kimpimaki T, Kulmala P, Savola K et al (2002) Natural history of beta-cell autoimmunity in young children with increased genetic susceptibility to type 1 diabetes recruited from the general population. J Clin Endocrinol Metab 87:4572-4579

7. Parikka V, Nanto-Salonen K, Saarinen M et al (2012) Early seroconversion and rapidly increasing autoantibody concentrations predict prepubertal manifestation of type 1 diabetes in children at genetic risk. Diabetologia 55:1926-1936

8. Ziegler AG, Hummel M, Schenker M, Bonifacio E (1999) Autoantibody appearance and risk for development of childhood diabetes in offspring of parents with type 1 diabetes: the 2-year analysis of the German BABYDIAB Study. Diabetes 48:460-468

9. Bingley PJ (2010) Clinical applications of diabetes antibody testing. J Clin Endocrinol Metab 95:25-33

10. Ziegler AG, Rewers M, Simell O et al (2013) Seroconversion to multiple islet autoantibodies and risk of progression to diabetes in children. JAMA 309:2473-2479

11. Howson JM, Stevens H, Smyth DJ et al (2011) Evidence that HLA class I and II associations with type 1 diabetes, autoantibodies to GAD and autoantibodies to IA-2, are distinct. Diabetes 60:2635-2644

12. Achenbach P, Warncke K, Reiter J et al (2004) Stratification of type 1 diabetes risk on the basis of islet autoantibody characteristics. Diabetes 53:384-392

13. Hoppu S, Ronkainen MS, Kulmala P, Akerblom HK, Knip M (2004) GAD65 antibody isotypes and epitope recognition during the prediabetic process in siblings of children with type I diabetes. Clin Exp Immunol 136:120-128

14. Mayr A, Schlosser M, Grober N et al (2007) GAD autoantibody affinity and epitope specificity identify distinct immunization profiles in children at risk for type 1 diabetes. Diabetes 56:1527-1533

15. Pihoker C, Gilliam LK, Hampe CS, Lernmark A (2005) Autoantibodies in diabetes. Diabetes 54(Suppl 2):S52-S61

16. Ronkainen MS, Hoppu S, Korhonen S et al (2006) Early epitope- and isotype-specific humoral immune responses to GAD65 in young children with genetic susceptibility to type 1 diabetes. Eur J Endocrinol 155:633-642

17. Bonifacio E, Lampasona V, Bernasconi L, Ziegler AG (2000) Maturation of the humoral autoimmune response to epitopes of GAD in preclinical childhood type 1 diabetes. Diabetes 49:202-208

18. Bingley PJ, Bonifacio E, Williams AJ, Genovese S, Bottazzo GF, Gale EA (1997) Prediction of IDDM in the general population: strategies based on combinations of autoantibody markers. Diabetes 46:1701-1710 
19. Douek IF, Gillespie KM, Bingley PJ, Gale EA (2002) Diabetes in the parents of children with Type I diabetes. Diabetologia 45:495-501

20. Strebelow M, Schlosser M, Ziegler B, Rjasanowski I, Ziegler M (1999) Karlsburg type I diabetes risk study of a general population: frequencies and interactions of the four major type I diabetesassociated autoantibodies studied in 9419 schoolchildren. Diabetologia 42:661-670

21. Schlosser M, Strebelow M, Rjasanowski I, Kerner W, Wassmuth R, Ziegler M (2004) Prevalence of diabetesassociated autoantibodies in schoolchildren: the Karlsburg Type 1 Diabetes Risk Study. Ann N Y Acad Sci 1037:114-117

22. Schlosser M, Strebelow M, Wassmuth R et al (2002) The Karlsburg type 1 diabetes risk study of a normal schoolchild population: association of beta-cell autoantibodies and human leukocyte antigen-DQB1 alleles in antibody-positive individuals. J Clin Endocrinol Metab 87:2254-2261

23. Torn C, Mueller PW, Schlosser M, Bonifacio E, Bingley PJ, Participating L (2008) Diabetes Antibody Standardization Program: evaluation of assays for autoantibodies to glutamic acid decarboxylase and islet antigen-2. Diabetologia 51:846-852

24. Schlosser M, Mueller PW, Torn C, Bonifacio E, Bingley PJ (2010) Diabetes Antibody Standardization Program: evaluation of assays for insulin autoantibodies. Diabetologia 53:2611-2620

25. Curnock RM, Reed CR, Rokni S, Broadhurst JW, Bingley PJ, Williams AJ (2012) Insulin autoantibody affinity measurement using a single concentration of unlabelled insulin competitor discriminates risk in relatives of patients with type 1 diabetes. Clin Exp Immunol 167:67-72
26. Schlosser M, Koczwara K, Kenk H et al (2005) In insulin-autoantibodypositive children from the general population, antibody affinity identifies those at high and low risk. Diabetologia 48:1830-1832

27. Achenbach P, Koczwara K, Knopff A, Naserke H, Ziegler AG, Bonifacio E (2004) Mature high-affinity immune responses to (pro)insulin anticipate the autoimmune cascade that leads to type 1 diabetes. J Clin Investig 114:589-597

28. Siljander H, Harkonen T, Hermann R et al (2009) Role of insulin autoantibody affinity as a predictive marker for type 1 diabetes in young children with HLA-conferred disease susceptibility. Diabetes Metab Res Rev 25:615-622

29. Krause S, Chmiel R, Bonifacio E et al (2012) IA-2 autoantibody affinity in children at risk for type 1 diabetes. Clin Immunol 145:224-229

30. Achenbach P, Lampasona V, Landherr U et al (2009) Autoantibodies to zinc transporter 8 and SLC30A8 genotype stratify type 1 diabetes risk. Diabetologia 52:1881-1888

31. De Grijse J, Asanghanwa M, Nouthe B et al (2010) Predictive power of screening for antibodies against insulinoma-associated protein 2 beta (IA-2beta) and zinc transporter-8 to select first-degree relatives of type 1 diabetic patients with risk of rapid progression to clinical onset of the disease: implications for prevention trials. Diabetologia 53:517-524

32. Yu L, Boulware DC, Beam CA et al (2012) Zinc transporter-8 autoantibodies improve prediction of type 1 diabetes in relatives positive for the standard biochemical autoantibodies. Diabetes Care 35:1213-1218

33. Miao D, Guyer KM, Dong F et al (2013) GAD65 autoantibodies detected by electrochemiluminescence assay identify high risk for type 1 diabetes. Diabetes 62:4174-4178 